\title{
Boundary integral equations for the exterior Robin problem in two dimensions
}

\author{
Olha Ivanyshyn Yaman*, Gazi Özdemir \\ Department of Mathematics, Izmir Institute of Technology, Urla, Izmir 35430, Turkey
}

\section{A R T I C L E I N F O}

\section{MSC:}

$35 \mathrm{~J} 25$

$31 \mathrm{~A} 10$

45B05

65 N35

\section{Keywords:}

Exterior Robin problem

Boundary integral equations

Laplace equation

\begin{abstract}
A B S T R A C T
We propose two methods based on boundary integral equations for the numerical solution of the planar exterior Robin boundary value problem for the Laplacian in a multiply connected domain. The methods do not require any a-priori information on the logarithmic capacity. Investigating the properties of the integral operators and employing the Riesz theory we prove that the obtained boundary integral equations for both methods are uniquely solvable. The feasibility of the numerical methods is illustrated by examples obtained via solving the integral equations by the Nyström method based on weighted trigonometric quadratures on an equidistant mesh.
\end{abstract}

(c) 2018 Elsevier Inc. All rights reserved.

\section{Introduction}

The Laplace equation arises in many areas in physics and mathematics, such as electromagnetism, fluid mechanics, heat conduction, geometry etc. The Robin or impedance condition models the situation when the boundary absorbs some part of the energy, heat, mass, which is transmitted through it. Mathematically, the problem can be stated as follows. Let $D$ be a bounded domain in $\mathbb{R}^{2}$ with a smooth boundary $\Gamma$. By $v$ we denote the unit normal to the boundary directed into the exterior of $D$. For brevity we denote by $D^{+}$the exterior of $D$, i.e. $D^{+}=\mathbb{R}^{2} \backslash \bar{D}$. The exterior Robin boundary value problem for the Laplace equation can be formulated as following. Given a function $f \in C(\Gamma), \lambda \in C(\Gamma), \lambda>0$ find a solution $u \in C^{2}\left(D^{+}\right) \cap C^{1}(\Gamma)$ of the Laplace equation

$$
\Delta u=0 \text { in } D^{+}
$$

that satisfies the impedance boundary condition

$$
\frac{\partial u}{\partial v}-\lambda u=f \quad \text { on } \Gamma
$$

In addition, the solution to the exterior problem should satisfy the asymptotic behavior at infinity, i.e.

$$
u(x)=O(1), \quad|x| \rightarrow \infty .
$$

It is well-known that the problem has a unique solution, [1-3]. In particular, [1], the unique solution $u$ to (1.1)-(1.3) has the following asymptotic behavior

$$
u(x)=\omega+O\left(\frac{1}{|x|}\right), \quad|x| \rightarrow \infty, \quad \omega=\text { const }
$$

\footnotetext{
* Corresponding author. Tel.

E-mail address: olhaivanyshyn@iyte.edu.tr (O. Ivanyshyn Yaman).
} 
and

$$
\frac{\partial u}{\partial|x|}(x)=O\left(\frac{1}{|x|^{2}}\right), \quad|x| \rightarrow \infty .
$$

Provided the domain $D$ is simply-connected and contains the origin the exterior Robin problem can be converted to an equivalent interior boundary value problem via the Kelvin transform.

In this paper we are interested in the numerical solution methods of the problem (1.1)-(1.3) for multiply-connected domains via boundary integral equation method. Although there is a vast number of publication on the Dirichlet and Neumannn problem [4-6], there are only few studies for the Robin boundary value problem in two dimensions, e.g. the study of perturbed half-space impedance problem [7]. For the exterior Robin problem we consider to seek the solution in the form of single-layer potential in order to avoid an operator with a hypersingular kernel. However, there are two aspects that should be taken care of: the single-layer potential has a logarithmic grows and, moreover, there exist pathological boundaries, such that logarithmic capacity of the curve is equal one. Representation of the solution to (1.1)-(1.3) in terms of the classical single-layer potential is justified only under the additional condition on the unknown density, which makes this approach hard for numerical solution. Constanda, [1], suggested a modification to the single layer potential without additional restrictions of the unknown function but a-priori knowledge of the logarithmic capacity is required. Inspired by the modifications of the single-layer potential which were designed for the Dirichlet problem, [6] and for inverse problem, [8], we propose their application to the solution of the exterior Robin problem. Main advantage of the modifications is that they deal both with the issues of pathological boundaries and with unboudedness of the solution at infinity, without any further assumptions.

\section{Boundary integral equations}

Recalling the fundamental solution to the Laplace equation in two dimensions

$$
\Phi(x, y)=\frac{1}{2 \pi} \ln \frac{1}{|x-y|}, x \neq y
$$

one can see that the single layer potential

$$
u(x)=\int_{\Gamma} \Phi(x, y) \varphi(y) d s(y), x \in D^{+}, \varphi \in C(\Gamma)
$$

does not satisfy the asymptotic behavior (1.3) at infinity unless $\int_{\Gamma} \varphi d s=0$. To remove this condition on the unknown density we propose two modifications. The first one is to apply a modified single-layer potential, proposed for the Dirichlet problem in [6, p. 134]. We define the mean value operator

$$
M: \varphi \mapsto \frac{1}{|\Gamma|} \int_{\Gamma} \varphi d s
$$

and introduce the modified single-layer potential

$$
u(x)=\int_{\Gamma} \Phi(x, y)(\psi(y)-M \psi) d s(y)+M \psi, \quad x \in \mathbb{R}^{2} \backslash \Gamma
$$

The second approach which we proposed is based on the modification of the kernel of the single-layer potential, firstly used by [8] in the context of an inverse problem,

$$
u(x)=\int_{\Gamma}\left[\Phi(x, y)-\Phi\left(x, x^{\diamond}\right)\right] \psi(y) d s(y)+M \psi, x \in \mathbb{R}^{2} \backslash \Gamma
$$

where $x^{\diamond} \in D$. From the asymptotic behavior of the fundamental solution

$$
\Phi(x, y)=\frac{1}{2 \pi} \ln \frac{1}{|x|}+O\left(\frac{1}{|x|}\right), \quad|x| \rightarrow \infty
$$

we can see that both of these modifications ensure boundedness of the function $u$ at infinity and we can seek the solution to (1.1)-(1.3) either in the form (2.2) or (2.3). Substituting (2.2) and (2.3) to the boundary condition (1.2) in view of the jump relations for the single-layer potential, [6], we obtain the following Fredholm integral equations of the second kind

$$
-\frac{1}{2}(\psi(x)-M \psi)+\int_{\Gamma}\left(\frac{\partial \Phi(x, y)}{\partial \nu(x)}-\lambda(x) \Phi(x, y)\right)(\psi(y)-M \psi) d s(y)-\lambda(x) M \psi=f(x), x \in \Gamma
$$

and

$$
\begin{aligned}
& -\frac{1}{2} \psi(x)+\int_{\Gamma}\left(\frac{\partial \Phi(x, y)}{\partial \nu(x)}-\lambda(x) \Phi(x, y)\right) \psi(y) d s(y) \\
& -\int_{\Gamma} \frac{\partial \Phi\left(x, x^{\diamond}\right)}{\partial v(x)} \psi(y) d s(y)+\lambda(x) \int_{\Gamma} \Phi\left(x, x^{\diamond}\right) \psi(y) d s(y)-\lambda(x) M \psi=f(x), x \in \Gamma
\end{aligned}
$$


which are equivalent to the boundary value problem (1.1)-(1.3). We note that due to the representations (2.2) and (2.3), not only the solution $u$ can be found at any point but also its asymptotics, the constant $\omega$ in (1.4), which is given by $M \psi$.

To prove the unique solvability of the integral equation (2.4) and (2.5) we introduce the following linear boundary integral operators defined for $\psi \in C(\Gamma)$

$$
\begin{aligned}
& (S \psi)(x)=2 \int_{\Gamma} \Phi(x, y) \psi(y) d s(y), x \in \Gamma \\
& \left(K^{\prime} \psi\right)(x)=2 \int_{\Gamma} \frac{\partial \Phi(x, y)}{\partial v(x)} \psi(y) d s(y), x \in \Gamma
\end{aligned}
$$

Theorem 2.1. The integral equation (2.4) or equivalently

$$
\left(\left(I-K^{\prime}+\lambda S\right)(I-M)+2 \lambda M\right) \psi=-2 f
$$

has a unique solution $\psi \in C(\Gamma)$ for any $f \in C(\Gamma), \lambda \in C(\Gamma), \lambda>0$.

Proof. Since the kernels of the integral operators $S, K^{\prime}, M$ are weakly singular or continuous, the operators are compact from $C(\Gamma)$ to $C(\Gamma)$. Hence, we have the operator equation of the second kind $(I+A) \psi=0$, where $A$ is compact. Due to the Riesz theorem it is sufficient to show that the corresponding homogeneous equation has only the trivial solution.

Introducing the function

$$
u(x)=\int_{\Gamma} \Phi(x, y)(\psi(y)-M \psi) d s(y)+M \psi, \quad x \in D^{+},
$$

where $\psi$ is a solution to the homogeneous version of (2.6) we observe that $u$ is a solution to the exterior Robin boundary value problem and by uniqueness $u \equiv 0$ in $D^{+}$. Since $u$ is continuous up to the boundary we have $u=0$ on $\Gamma$, i.e.

$$
(S-S M) \psi+2 M \psi=0
$$

The operator $(S-S M+2 M): C(\Gamma) \rightarrow C(\Gamma)$ is injective, [6, Theorem 7.41], i.e. $\psi=0$.

Theorem 2.2. The integral equation (2.5) or equivalently

$$
\psi-\left(K^{\prime}-\lambda S-2|\Gamma|\left(\frac{\partial \Phi\left(\cdot, x^{\diamond}\right)}{\partial v}-\lambda\left(1 /|\Gamma|-\Phi\left(\cdot, x^{\diamond}\right)\right)\right) M\right) \psi=-2 f
$$

has a unique solution $\psi \in C(\Gamma)$ for any $f \in C(\Gamma), \lambda \in C(\Gamma), \lambda>0$.

Proof. Similarly to the previous theorem we consider the corresponding homogenous integral equation. Introducing the modified single-layer potential (2.3) by the uniqueness of the exterior Robin boundary value problem, we obtain

$$
0=\int_{\Gamma}\left[\Phi(x, y)-\Phi\left(x, x^{\diamond}\right)\right] \psi(y) d s(y)+M \psi, x \rightarrow \infty,
$$

that is $\int_{\Gamma} \psi d s$ has to vanish. From Green's theorem we have

$$
0=\int_{D^{+}}|\nabla u|^{2} d x=-\int_{\Gamma} \lambda\left(\frac{\partial u}{\partial v}\right)^{2} d s \leq 0
$$

and hence $\left.\frac{\partial u}{\partial v}\right|_{\Gamma}=0$, which together with $\int_{\Gamma} \psi d s=0$ yields

$$
\left(-I+K^{\prime}\right) \psi=0
$$

Since $\left(I-K^{\prime}\right)$ has a trivial nullspace, [6, Theorem 6.21], $\psi=0$.

Theorems 2.1 and 2.2 combined with Riesz's theory guarantee applicability of the proposed methods for a positive impedance function $\lambda$ and continuous boundary data. In order to investigate the limit case scenarios, i.e. $\lambda \rightarrow 0$ and $\lambda \rightarrow \infty$, we prove the following lemmas.

Lemma 2.1. The integral equation

$$
\psi-\left(K^{\prime}-2|\Gamma| \frac{\partial \Phi\left(\cdot, x^{\diamond}\right)}{\partial v} M\right) \psi=0, \quad x^{\diamond} \in D
$$

has a nontrivial solution.

Proof. The adjoint integral equation reads

$$
((I-K) \varphi)(x)+2(K \varphi)\left(x^{\triangleright}\right)=0
$$


Choosing $\varphi \equiv 1$ and using the fact that

$$
2 \int_{\partial D} \frac{\partial \Phi(x, y)}{\partial v(y)} d s(y)= \begin{cases}-2, & x \in D \\ -1, & x \in \partial D \\ 0, & x \in D^{+}\end{cases}
$$

we find out that the adjoint equation has a nontrivial solution. The statement follows from the Fredholm alternative.

Lemma 2.2. The integral operators $S_{0}: C^{0, \alpha}(\Gamma) \rightarrow C^{1, \alpha}(\Gamma)$ and $S_{1}: C^{0, \alpha}(\Gamma) \rightarrow C^{1, \alpha}(\Gamma)$ defined by

$$
S_{0}=(S-S M+2 M), \quad S_{1}:=\left(S+2 M-|\Gamma| \Phi\left(\cdot, x^{\diamond}\right) M\right), \quad x^{\diamond} \in D
$$

are bijective.

Proof. The operator $S_{0}=(S-S M+2 M): C^{0, \alpha}(\Gamma) \rightarrow C^{1, \alpha}(\Gamma)$ is bijective, [6, Theorem 7.41]. Analogously to the proof of Theorem 2.2 it can be shown that the operator $S_{1}$ is injective. Rewriting $S_{1}$ in the form

$$
S_{1}=S_{0}+S M-|\Gamma| \Phi\left(\cdot, x^{\diamond}\right) M=S_{0}\left(I+S_{0}^{-1}\left(S-|\Gamma| \Phi\left(\cdot, x^{\diamond}\right)\right) M\right)
$$

we obtain that $I+S_{0}^{-1}\left(S-|\Gamma| \Phi\left(\cdot, x^{\diamond}\right)\right) M$ is injective. Furthermore, by Banach open mapping theorem $S_{0}^{-1}$ is bounded. The operator $\left(S-|\Gamma| \Phi\left(\cdot, x^{\diamond}\right)\right) M: C^{0, \alpha}(\Gamma) \rightarrow C^{0, \alpha}(\Gamma)$ is compact and hence by the Riesz theory the operator $S_{1}$ is bijective.

Due to the fact that $(I-M)$ const $=0$ and Lemma 2.1 both homogeneous integral equation (2.6) and (2.8) have nontrivial solutions for $\lambda=0$. Therefore, in the case of impedance function $\lambda$ of very small magnitude, the fully discrete versions of the proposed methods will have the burden of increasing condition number and a slow convergence.

In the case of extremely big magnitude impedance function $\lambda$, Lemma 2.2 ensures that, under the restriction of Lipschitz continuous differentiability of the boundary data and impedance function, the numerical solutions of the integral equations (2.6) and (2.8) are stable and of the same order of convergence as for the average magnitude impedance function $\lambda$.

\section{Numerical solution}

For the numerical solution of the Fredholm integral equations of the second kind (2.6) and (2.8) we apply Nyström's method. We assume that the boundary curves are parametrized in the form

$$
\Gamma=\{z(t): t \in[0,2 \pi]\},
$$

where $z: \mathbb{R} \rightarrow \mathbb{R}^{2}$ are $2 \pi$ periodic, twice continuously differentiable and injective functions, i.e. $\left|z^{\prime}(t)\right| \neq 0$ for all $t \in[0,2 \pi]$. In addition, we assume that the orientation of $\Gamma$, is counter-clockwise and define an outward unit normal vector to the boundary $\Gamma$ by $v=\left(z_{2}^{\prime},-z_{1}^{\prime}\right) /\left|z^{\prime}\right|$.

In the numerical scheme two types of integral operators occur, the integral operators with smooth and with weaklysingular kernels. For the numerical integration of smooth functions and integral operator $K_{n}^{\prime}$ with continuous kernel we apply a composite trapezoidal quadrature rule.

Numerical approximation of integral operators with the weakly singular kernels can be performed either via a global type rule such as hybrid Gauss-trapezoidal quadrature, [9], Kapur-Rokhlin quadrature, [10] etc. or panel-based type quadrature rule, e.g. Helsing and Modified Gaussian (Kolm-Rokhlin). For the overview and comparison of these quadrature rule we refer to [11]. Since all functions in the integral equations are $2 \pi$ periodic, due to the parameterization (3.1), we chose the method of splitting off the singularity in the kernel of integral operator, [6,12]. The kernel is presented as a sum of a singular part which can be integrated analytically and a smooth part

$$
2 \pi \Phi(z(t), z(\tau))=-\ln \left|4 \sin ^{2} \frac{t-\tau}{2}\right|+\ln \frac{\left|4 \sin ^{2} \frac{t-\tau}{2}\right|}{|z(t)-z(\tau)|^{2}},
$$

with the limit values $-\ln \left|z^{\prime}(t)^{2}\right|$ as $\tau \rightarrow t$. For the improper integral we use a quadrature formula due to [6,13], which is uniformly convergent for all trigonometric polynomials

$$
\frac{1}{2 \pi} \int_{0}^{2 \pi} \ln \left(4 \sin ^{2} \frac{t-\tau}{2}\right) \psi(\tau) d \tau \approx \sum_{j=0}^{2 n-1} R_{j}^{(n)}(t) \psi\left(t_{j}\right),
$$

where

$$
R_{j}^{(n)}(t)=-\frac{1}{n}\left\{\sum_{m=1}^{n-1} \frac{1}{m} \cos \left(m\left(t-t_{j}\right)\right)+\frac{1}{2 n} \cos \left(n\left(t-t_{j}\right)\right)\right\},
$$

with equidistant points $t_{j}=\frac{j \pi}{n}$ for $j=0, \ldots, 2 n-1$.

In the case of analytic functions the quadrature error is exponentially decreasing. Furthermore, provided that the kernels of integral operators, right-hand side, impedance function and exact solution to the integral equation (2.6), (2.8) are analytic, the error for the approximate solution is also exponentially decreasing. 
Table 1

Error analysis of both methods for $\Gamma$ a unit circle.

\begin{tabular}{lllll}
\hline$n$ & cond 1 & $\left\|u-u^{\dagger}\right\|$ & cond2 & $\left\|u-u^{\dagger}\right\|$ \\
\hline 16 & $1.9 \mathrm{e}+00$ & $8.9 \mathrm{e}-16$ & $1.9 \mathrm{e}+00$ & $1.8 \mathrm{e}-15$ \\
32 & $1.9 \mathrm{e}+00$ & $8.9 \mathrm{e}-16$ & $1.9 \mathrm{e}+00$ & $1.8 \mathrm{e}-15$ \\
64 & $1.9 \mathrm{e}+00$ & $1.8 \mathrm{e}-15$ & $2.0 \mathrm{e}+00$ & $2.7 \mathrm{e}-15$ \\
\hline
\end{tabular}

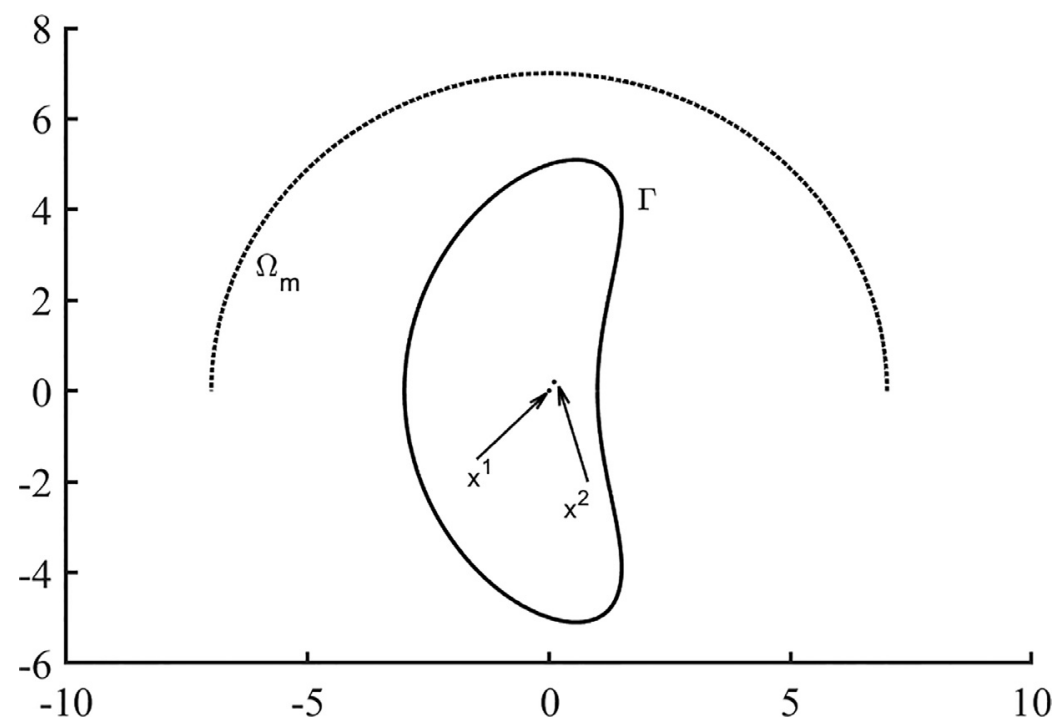

Fig. 1. $\Gamma$ with measurement curve.

\section{Numerical examples}

For the function

$$
f(x)=\frac{\partial\left(\Phi\left(x, x^{1}\right)-\Phi\left(x, x^{2}\right)\right)}{\partial \nu(x)}-\lambda(x)\left(C+\Phi\left(x, x^{1}\right)-\Phi\left(x, x^{2}\right)\right), x^{1}, x^{2} \in D,
$$

the exact solution to the Robin exterior boundary value problem (1.1)-(1.3) is given by

$$
u^{\dagger}(x)=C+\Phi\left(x, x^{1}\right)-\Phi\left(x, x^{2}\right), x \in D^{+} .
$$

Using this exact solution $u \dagger$ with $x^{1}=(0,0), x^{2}=(0.1,0.2)$ and $C=5$ we present numerical examples which illustrate the accuracy and stability of the proposed method.

In the first example we choose $\lambda \equiv 1$ and test our method for the case of a pathological boundary, $\int_{\Gamma} \Phi(x, y) d s(y)=0$, i.e. we consider the domain $D$ to be a unit disc. Both schemes are constructed to be independent of the logarithmic potential of the boundary and this is also verified by the numerical results in Table 1 where $\mathrm{n}$ is a discretization parameter. For all examples the interior point is chosen as $x^{\diamond}=(0,0)$. By $\left\|u-u^{\dagger}\right\|$ we denote the absolute maximum error of the numerical solution over curve $\Omega_{m}=\{z(t)=(7 \cos t, 7 \sin t) \quad t \in[0,2 \pi]\}$ and by cond 1 and cond 2 we label the condition numbers of the matrix of the fully discretized integral equations (2.6) and (2.8), correspondingly.

In the next group of examples we consider the boundary

$$
\Gamma=\left\{z(t)=\left(2 \cos t-2 \cos ^{2} t+1,5 \sin t-\cos t \sin t\right), \quad t \in[0,2 \pi]\right\}
$$

depicted on Fig. 1 and the impedance function, see Fig. 2, either a Gaussian

$$
\lambda(t)=c_{\lambda}\left(e^{-\frac{(t-\pi)^{2}}{2}}+10^{-5}\right)
$$

or a piecewise linear function

$$
\lambda(t)=c_{\lambda}\left(\pi-|\pi / 2-t|-|3 \pi / 2-t|+1.2 \mid \pi-t+10^{-5}\right),
$$

where $c_{\lambda}$ is a scaling parameter.

Modifying the scaling parameter $c_{\lambda}$ we investigate stability and convergence of the algorithms for the Robin boundary condition and the limiting Dirichlet and Neumann case with very big and very small constant $c_{\lambda}$ correspondingly.

As can be seen from Table 2, in the case of $c_{\lambda}=1$ the methods converge super-algebraically and the condition numbers are uniformly bounded as it is expected for the Nyström method. 

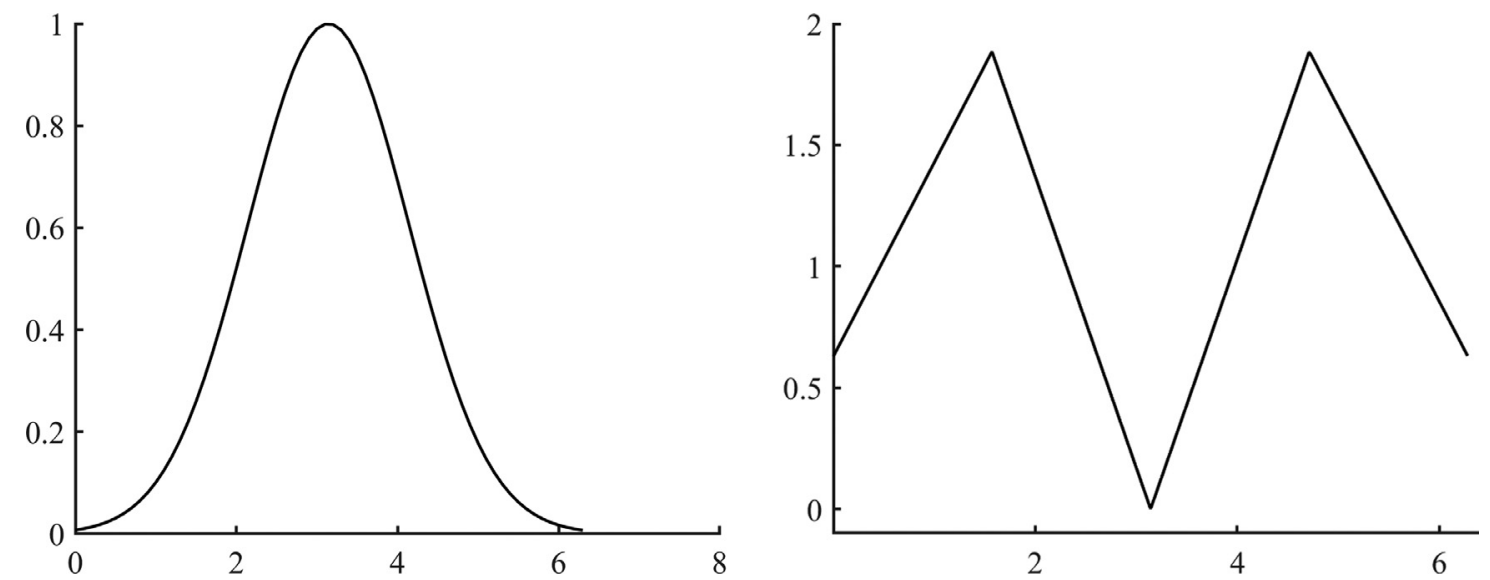

Fig. 2. Robin functions.

Table 2

Error analysis, $c_{\lambda}=1$

\begin{tabular}{|c|c|c|c|c|c|c|c|c|}
\hline \multirow[t]{2}{*}{$n$} & \multicolumn{4}{|l|}{ Gaussian } & \multicolumn{4}{|c|}{ Piecewise linear function } \\
\hline & cond1 & $\left\|u-u^{\dagger}\right\|$ & cond2 & $\left\|u-u^{\dagger}\right\|$ & cond 1 & $\left\|u-u^{\dagger}\right\|$ & cond2 & $\left\|u-u^{\dagger}\right\|$ \\
\hline 16 & $1.0 \mathrm{e}+01$ & $1.9 e-04$ & $1.8 \mathrm{e}+01$ & $3.1 \mathrm{e}-02$ & $8.9 e+00$ & $3.1 \mathrm{e}-05$ & $1.3 e+01$ & 5.1e-03 \\
\hline 32 & $9.9 e+00$ & 6.6e-07 & $1.8 \mathrm{e}+01$ & $1.6 e-05$ & $8.5 e+00$ & $1.1 \mathrm{e}-07$ & $1.3 e+01$ & 3.0e-06 \\
\hline 64 & $9.9 e+00$ & $3.9 e-13$ & $1.8 \mathrm{e}+01$ & $8.1 \mathrm{e}-12$ & $8.4 e+00$ & $5.3 e-13$ & $1.3 e+01$ & $1.4 \mathrm{e}-11$ \\
\hline 128 & $9.9 e+00$ & $2.4 \mathrm{e}-14$ & $1.8 \mathrm{e}+01$ & $8.9 e-15$ & $8.4 e+00$ & $1.6 e-14$ & $1.3 e+01$ & $2.7 e-15$ \\
\hline 256 & $9.9 e+00$ & 3.6e-15 & $1.8 \mathrm{e}+01$ & $5.3 e-15$ & $8.4 e+00$ & $3.6 e-15$ & $1.3 e+01$ & 8.0e-15 \\
\hline
\end{tabular}

Table 3

Error analysis, $c_{\lambda}=10^{10}$.

\begin{tabular}{|c|c|c|c|c|c|c|c|c|}
\hline \multirow[t]{2}{*}{$n$} & \multicolumn{4}{|l|}{ Gaussian } & \multicolumn{4}{|c|}{ Piecewise linear function } \\
\hline & cond 1 & $\left\|u-u^{\dagger}\right\|$ & cond2 & $\overline{\left\|u-u^{\dagger}\right\|}$ & cond1 & $\left\|u-u^{\dagger}\right\|$ & cond2 & $\left\|u-u^{\dagger}\right\|$ \\
\hline 16 & $9.2 \mathrm{e}+02$ & $3.0 \mathrm{e}-06$ & $9.3 e+02$ & $4.4 \mathrm{e}-04$ & $9.9 e+05$ & $3.0 \mathrm{e}-06$ & $1.0 \mathrm{e}+06$ & $4.4 \mathrm{e}-04$ \\
\hline 32 & $2.0 e+03$ & 4.7e-09 & $2.0 e+03$ & 1.1e-07 & $2.0 e+06$ & 4.7e-09 & $2.0 e+06$ & $1.1 \mathrm{e}-07$ \\
\hline 64 & $4.5 e+03$ & $3.6 e-14$ & $4.5 e+03$ & $2.5 e-14$ & $3.9 e+06$ & $2.0 \mathrm{e}-14$ & $3.9 e+06$ & $2.0 \mathrm{e}-14$ \\
\hline 128 & $9.5 e+03$ & $3.1 \mathrm{e}-13$ & $9.5 e+03$ & $8.0 e-15$ & $7.9 e+06$ & $3.4 \mathrm{e}-14$ & $7.9 e+06$ & $9.8 e-15$ \\
\hline 256 & $2.0 e+04$ & $6.1 \mathrm{e}-13$ & $2.0 e+04$ & 7.1e-15 & $1.6 e+07$ & $2.1 \mathrm{e}-12$ & $1.6 e+07$ & $2.2 \mathrm{e}-12$ \\
\hline
\end{tabular}

Table 4

Error analysis, $c_{\lambda}=10^{-3}$.

\begin{tabular}{|c|c|c|c|c|c|c|c|c|}
\hline \multirow[t]{2}{*}{$n$} & \multicolumn{4}{|l|}{ Gaussian } & \multicolumn{4}{|c|}{ Piecewise linear function } \\
\hline & cond 1 & $\left\|u-u^{\dagger}\right\|$ & cond2 & $\left\|u-u^{\dagger}\right\|$ & cond 1 & $\left\|u-u^{\dagger}\right\|$ & cond2 & $\left\|u-u^{\dagger}\right\|$ \\
\hline 16 & $1.7 e+03$ & $3.3 e-02$ & $2.8 \mathrm{e}+04$ & $4.2 \mathrm{e}+01$ & $8.3 e+02$ & $1.6 e-02$ & $4.3 e+03$ & $6.4 \mathrm{e}+00$ \\
\hline 32 & $1.7 e+03$ & $1.2 \mathrm{e}-04$ & $3.7 e+03$ & $3.0 \mathrm{e}-03$ & $8.3 e+02$ & 5.9e-05 & $1.9 e+03$ & $1.5 \mathrm{e}-03$ \\
\hline 64 & $1.7 e+03$ & $9.7 e-11$ & $3.7 e+03$ & $9.8 \mathrm{e}-10$ & $8.3 e+02$ & $4.7 \mathrm{e}-11$ & $1.9 e+03$ & $4.9 \mathrm{e}-10$ \\
\hline 128 & $1.7 e+03$ & $6.7 e-12$ & $3.7 e+03$ & $2.0 \mathrm{e}-12$ & $8.3 e+02$ & $3.2 \mathrm{e}-12$ & $1.9 e+03$ & $9.2 \mathrm{e}-13$ \\
\hline 256 & $1.7 e+03$ & $1.8 \mathrm{e}-12$ & $3.7 e+03$ & 1.6e-12 & $8.3 e+02$ & $9.4 \mathrm{e}-13$ & $1.9 e+03$ & 7.9e-13 \\
\hline
\end{tabular}

Increasing $c_{\lambda}$ leads to the growth of the condition number with the increase of the discretization parameter $n$, Table 3 . The reason for this lies in the fact that our schemes approaches the collocation method for integral equation of the first kind, the condition number of the linear system for which is not necessarily uniformly bounded on the contrary to Nyström's method.

As noted in Section 2, the integral equation for both methods have a non unique solution in the case $\lambda=0$. Therefore, we observe the expected increase in the condition numbers and decrease in the speed of convergence in the case of small $c_{\lambda}$, Tables 4 and 5 .

Having tested the feasibility of the methods, we now present a numerical solution of the electrostatic problem with the total field created by unit electric charges: monopole $\Phi\left(x^{\star}, \cdot\right)$ and dipole $\Phi\left(x_{1}^{\star}, \cdot\right)-\Phi\left(x_{2}^{\star}, \cdot\right)$, where $x^{\star}, x_{1}^{\star}, x_{2}^{\star} \in \mathbb{R}^{2} \backslash \bar{D}$. The 
Table 5

Error analysis, $c_{\lambda}=10^{-10}$.

\begin{tabular}{|c|c|c|c|c|c|c|c|c|}
\hline \multirow[t]{2}{*}{$n$} & \multicolumn{4}{|l|}{ Gaussian } & \multicolumn{4}{|c|}{ Piecewise linear function } \\
\hline & cond1 & $\left\|u-u^{\dagger}\right\|$ & cond2 & $\overline{\left\|u-u^{\dagger}\right\|}$ & cond1 & $\left\|u-u^{\dagger}\right\|$ & cond2 & $\left\|u-u^{\dagger}\right\|$ \\
\hline 16 & $1.7 e+10$ & $3.3 e+05$ & $3.3 e+03$ & $5.0 e+00$ & $8.3 e+09$ & $1.6 e+05$ & $3.3 e+03$ & $5.0 \mathrm{e}+00$ \\
\hline 32 & $1.7 e+10$ & $1.2 \mathrm{e}+03$ & $6.1 \mathrm{e}+06$ & $4.8 \mathrm{e}+00$ & $8.3 e+09$ & $5.9 e+02$ & $6.1 \mathrm{e}+06$ & $4.8 \mathrm{e}+00$ \\
\hline 64 & $1.7 e+10$ & $9.6 e-04$ & $3.7 e+10$ & $9.7 e-03$ & $8.3 e+09$ & $4.8 \mathrm{e}-04$ & $1.8 \mathrm{e}+10$ & $4.8 \mathrm{e}-03$ \\
\hline 128 & $1.7 e+10$ & $6.5 \mathrm{e}-05$ & $3.7 e+10$ & $1.9 \mathrm{e}-05$ & $8.3 e+09$ & 3.3e-05 & $1.8 \mathrm{e}+10$ & $9.1 \mathrm{e}-06$ \\
\hline 256 & $1.7 e+10$ & $1.4 \mathrm{e}-05$ & $3.7 e+10$ & $1.4 \mathrm{e}-05$ & $8.3 e+09$ & $9.3 e-06$ & $1.8 \mathrm{e}+10$ & 7.7e-06 \\
\hline
\end{tabular}
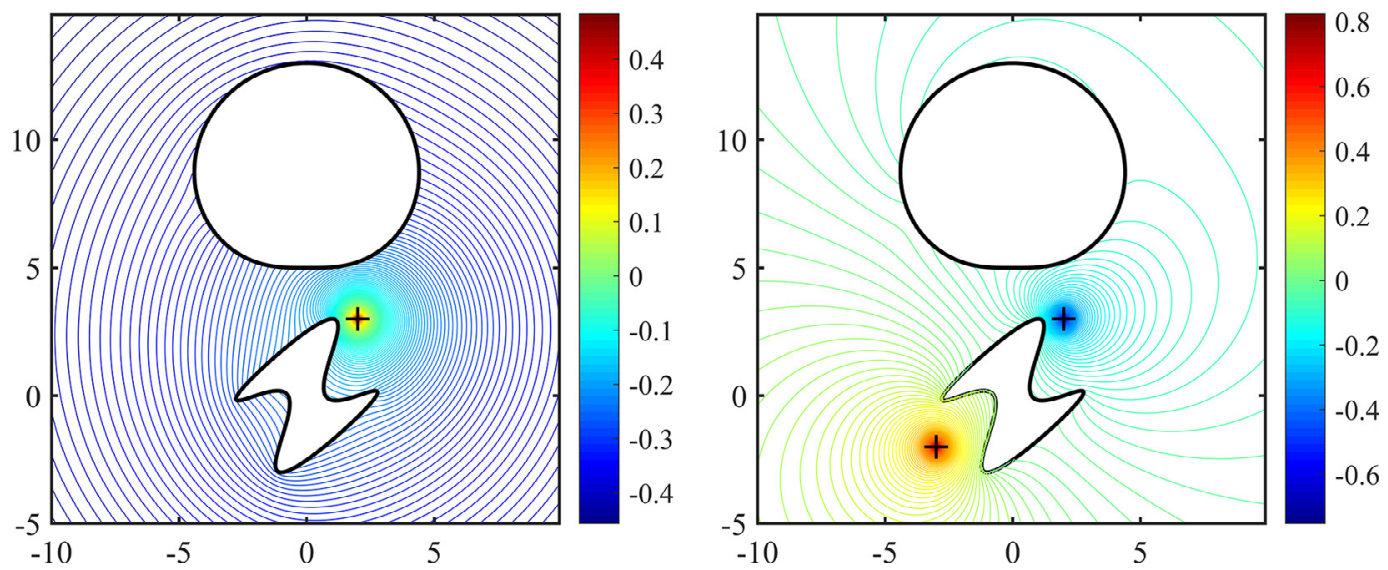

Fig. 3. Total field for a monopole and dipole, $c_{\lambda}=1$.

source points are taken as $x^{\star}=(2,3)$ for a monopole and $x_{1}^{\star}=(-3,-2), x_{2}^{\star}=(2,3)$ for a dipole. The total field $u^{T}$ for the monopole is constructed as follows

$$
u^{T}(x)=\Phi\left(x^{*}, x\right)+u(x), \quad x, x^{*} \in D^{+}
$$

where $u$ is the approximate solution to (1.1) and (1.3) obtained by the integral equation approach based on the representation (2.2) for the boundary data

$$
f(x)=-\left(\frac{\partial \Phi\left(x^{\star}, x\right)}{\partial \nu(x)}-\lambda(x) \Phi\left(x^{\star}, x\right)\right), x \in \Gamma, x^{\star} \in D^{+} .
$$

The total field $u^{T}$ for the dipole is given by

$$
u^{T}(x)=\Phi\left(x_{1}^{*}, x\right)-\Phi\left(x_{2}^{*}, x\right)+u(x), x_{1}^{*}, x_{2}^{*}, x \in D^{+}
$$

with the boundary data

$$
f(x)=-\left(\frac{\partial\left(\Phi\left(x_{1}^{\star}, x\right)-\Phi\left(x_{2}^{\star}, x\right)\right)}{\partial \nu(x)}-\lambda(x)\left(\Phi\left(x_{1}^{\star}, x\right)-\Phi\left(\left(x_{2}^{\star}, x\right)\right)\right), x \in \Gamma .\right.
$$

The domain $D$ consists of two simply connected domains bounded by the boundary

$$
\Gamma_{1}=\left\{z(t)=\left(4 \cos t+\sin 2 t, 4 \sin t+2 \sin ^{2} t+7\right), \quad t \in[0,2 \pi]\right\}
$$

and

$$
\Gamma_{2}=\{z(t)=(2 \cos t-\sin 3 t, 2 \sin t-\sin 3 t), \quad t \in[0,2 \pi]\} .
$$

The impedance function is chosen to be a piecewise linear function (4.3) and the discretization parameter in the Nyström method is $n=64$. The equipotential lines of total field for a monopole and dipole for the impedance function of average magnitude, i.e. $c_{\lambda}=1$, are presented in Fig. 3. In the next example, Fig. 4, we consider a case which approximates the Neumann boundary condition, by scaling the impedance function with $c_{\lambda}=10^{-3}$. The last example, Fig. 5, is dedicated to the limiting case scenario - Dirichlet boundary condition via choosing an impedance function of a large magnitude. 

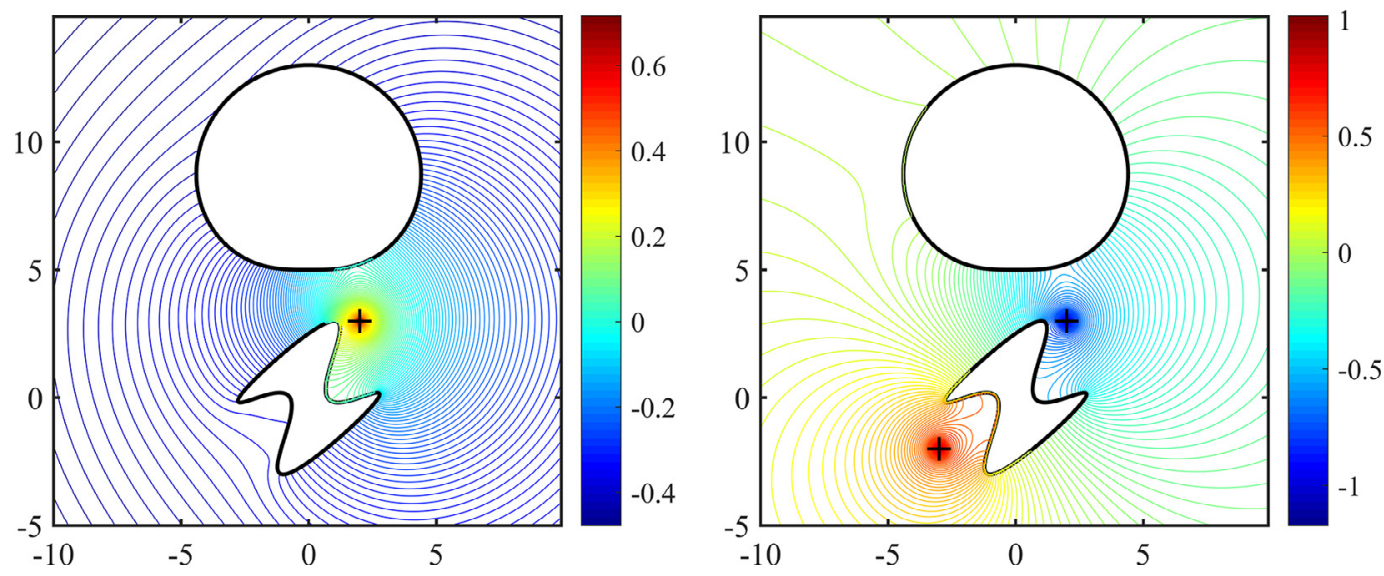

Fig. 4. Total field for a monopole and dipole, $c_{\lambda}=10^{-3}$.
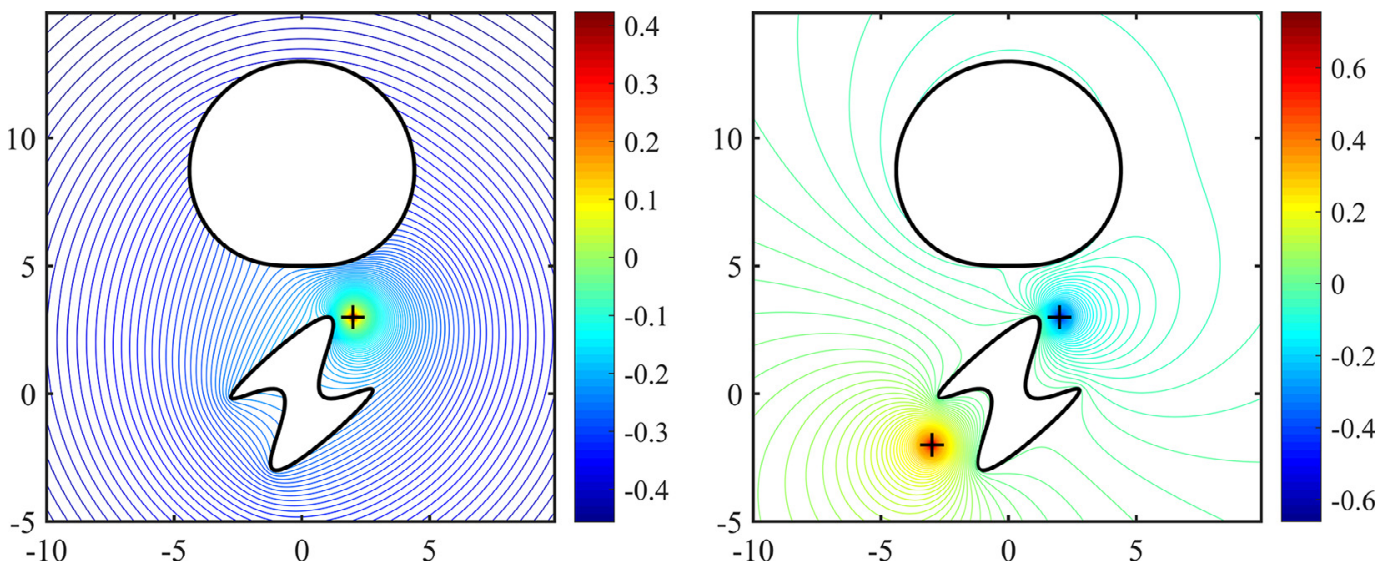

Fig. 5. Total field for a monopole and dipole, $c_{\lambda}=10^{10}$.

\section{Conclusion}

For the numerical solution of the exterior Robin boundary value problem in multiply connected domain we propose two integral equation methods which do not require any a priori information on the logarithmic capacity of the boundary. Both methods share the same properties such as super-algebraic convergence for analytic data and stability with respect to increase of the discretization parameter. The computational cost of the first method is slightly higher however it provides more accurate results for small discretization parameters. The methods are investigated also for the limit case scenarios $\lambda \rightarrow 0$ and $\lambda \rightarrow \infty$. In the case of Neumann boundary condition the derived integral equations are not uniquely solvable and therefore for a very small $\lambda$ the methods are not stable. On the contrary, in the case of Dirichlet boundary condition (very big $\lambda$ ) the integral equations are shown to be uniquely solvable and the proposed schemes provide numerical solutions with high convergence order although the condition numbers grow with increase of the discretization parameter.

\section{References}

[1] C. Constanda, Direct and Indirect Boundary Integral Equation Methods, Chapman \& Hall/CRC, Boca Raton, FL, 2000.

[2] C. Miranda, Partial Differential Equations of Elliptic Type, Springer-Verlag, New York-Berlin, 1970.

[3] D. Gilbarg, N.S. Trudinger, Elliptic partial differential equations of second order, Classics in Mathematics, Springer-Verlag, Berlin, 2001.

[4] K. Atkinson, Y. Jeon, Algorithm 788: automatic boundary integral equation programs for the planar Laplace equation., ACM Trans. Math. Softw. 24 (4) (1998) 395-417.

[5] J. Saavedra, J.D. Flores, Numerical solution of Laplace's equation on non-simply connected regions on IR²., Int. J. Comput. Math. 78 (2) (2001) 253-267.

[6] R. Kress, Linear integral equations, third, Springer, New York, 2014.

[7] R.O.H. Hoernig, Green's functions and integral equations for the Laplace and Helmholtz operators in impedance half-spaces, Ecole Polytechnique X, 2010 (Ph.D. thesis).

[8] R. Kress, W. Rundell, Nonlinear integral equations and the iterative solution for an inverse boundary value problem, Inverse Probl. 21 (4) (2005) 1207-1223.

[9] B.K. Alpert, Hybrid Gauss-trapezoidal quadrature rules, SIAM J. Sci. Comput. 20 (5) (1999) 1551-1584. (electronic) 
[10] S. Kapur, V. Rokhlin, High-order corrected trapezoidal quadrature rules for singular functions, SIAM J. Numer. Anal. 34 (4) (1997) 1331-1356.

[11] S. Hao, A.H. Barnett, P.G. Martinsson, P. Young, High-order accurate methods for Nyström discretization of integral equations on smooth curves in the plane, Adv. Comput. Math. 40 (1) (2014) 245-272.

[12] K.E. Atkinson, The Numerical solution of Integral Equations of the Second Kind., Cambridge University Press, Cambridge, 2009. Reprint of the 1997 hardback ed.

[13] K.E. Atkinson, A discrete Galerkin method for first kind integral equations with a logarithmic kernel, J. Integral Equ. Appl. 1 (3) (1988) 343-363. 